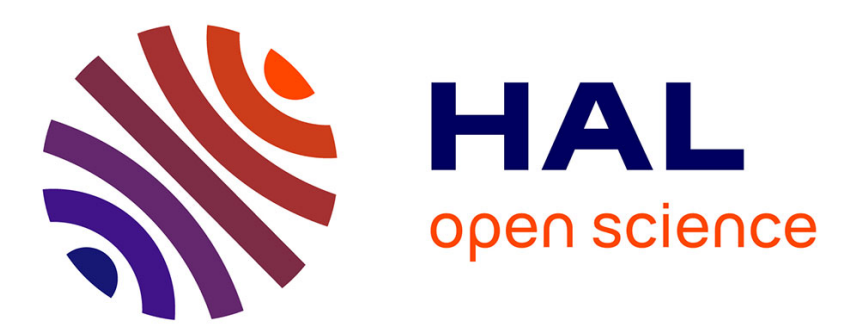

\title{
Finite-Time Position and Velocity Estimation Adapted to Noisy Biased Acceleration Measurements from Periodic Motion
}

\author{
Antonio Estrada, Denis Efimov, Wilfrid Perruquetti
}

\section{- To cite this version: \\ Antonio Estrada, Denis Efimov, Wilfrid Perruquetti. Finite-Time Position and Velocity Estimation Adapted to Noisy Biased Acceleration Measurements from Periodic Motion. International Journal of Control, 2016, 89 (9), 10.1080/00207179.2016.1175670 . hal-01295716}

\section{HAL Id: hal-01295716 https://inria.hal.science/hal-01295716}

Submitted on 31 Mar 2016

HAL is a multi-disciplinary open access archive for the deposit and dissemination of scientific research documents, whether they are published or not. The documents may come from teaching and research institutions in France or abroad, or from public or private research centers.
L'archive ouverte pluridisciplinaire HAL, est destinée au dépôt et à la diffusion de documents scientifiques de niveau recherche, publiés ou non, émanant des établissements d'enseignement et de recherche français ou étrangers, des laboratoires publics ou privés. 
To appear in the International Journal of Control

Vol. 00, No. 00, Month 20XX, 1-16

\title{
Finite-Time Position and Velocity Estimation Adapted to Noisy Biased Acceleration Measurements from Periodic Motion
}

\author{
Antonio Estrada, ${ }^{\text {a* }}$ Denis Efimov ${ }^{\mathrm{b}}$ and Wilfrid Perruquetti ${ }^{\mathrm{b}}$ \\ ${ }^{a}$ CONACYT Research Fellow - CIDESI, Playa Pie de la Cuesta 702, Col. Desarrollo San Pablo, 76125 \\ Queretaro, Mexico. \\ ${ }^{b}$ Non-A Team,Inria Lille - Nord Europe, Parc scientifique de la Haute Borne 40, avenue Halley - Bt A - \\ Park Plaza 59650 Villeneuve d’Ascq - France.Email: denis.efimov@inria.fr, wilfrid.perruquetti@inria.fr
}

(Received 00 Month 20XX; accepted 00 Month 20XX)

\begin{abstract}
The present work focuses on the problem of velocity and position estimation. A solution is presented for a class of oscillating systems in which position, velocity and acceleration are zero mean signals. The proposed scheme considers that the dynamic model of the system is unknown. Only noisy acceleration measurements, that may be contaminated by zero mean noise and constant bias, are considered to be available. The proposal uses the periodic nature of the signals obtaining finite-time estimations while tackling integration drift accumulation.
\end{abstract}

Keywords: Finite-time, estimation adaptation, periodic signals

\section{Introduction}

The estimation of position/velocity through the integration of measurements from inertial sensors inherently causes errors to grow with time, commonly known as integration drift. In fact, the process of zero-mean noise integration leads to an output that increases with integration time, even in the case that the accelerometer is at rest (Thong, Woolfson, Crowe, Hayes-Gill, \& Jones, 2004). For this reason, velocity/position estimation based on inertial sensors always requires some additional source of information providing for the corresponding correct signal value at some time instants. In general this information is updated with intervals significantly larger than the acceleration measurement rate. For instance, in inertial navigation systems (INS) aiding sensors are applied. An INS consists of a triad of orthogonal accelerometers and rate gyroscopes that are used to calculate both the translational and rotational movements of an aircraft over time (Rogers, 2003), a process know as dead reckoning. MEMS technology has made low-cost INS available. However, sensor biases coupled with the time integration, needed for dead reckoning, causes that the solution quickly diverges. That is why externally referenced sensors, such as the global positioning system, are required to regulate the error growth (Grewal, Weill, \& Andrew, 2007); Kalman filters are commonly used in order to fuse the two sources of information.

An alternative to the use of aiding sensors is the use of prior-knowledge of motion. In (Liu, Inoue, \& Shibata, 2009), for instance, the drift accumulation from gyroscope based orientation estimation, in human walking experiments, is compensated by detecting different gait phases with the aid of accelerometers and the knowledge of the human walking motion. In (Schepers, Asseldonk, Baten, \& Veltnik, 2010), foot placement for several strides is estimated. The drift is reduced applying the knowledge of the cyclic walking movement that leads to information on initial and final conditions. Another example of application of prior knowledge of motion is sensing and identification of physiological tremor. Tremor is defined as any involuntary, approximately rhythmic, and roughly sinusoidal movement [19]. In (Riviere, Ang, \& Khosla 2003), a filtering algorithm based on truncated Fourier series, so called weighted frequency Fourier linear combiner (WFLC), is applied for tremor identification. The WFLC models the tremor as a sinusoid and tracks its modulation in frequency, phase, and amplitude. The WFLC is suitable for signals with single dominant frequency, this 
restriction is relaxed in (Veluvolu \& Ang, 2011) by means of a band limited multiple Fourier linear combiner (BFMLC) which can be applied to band limited signals consisting of multiple components.

In the present work the prior-knowledge used in the velocity/position estimation solution proposal is periodicity of the motion. Therefore, a period estimation algorithm is fundamental considering that the oscillation period is in general not available. In (Spence \& Clarke, 2000), a method for estimating the period and amplitude profile of a signal with multiple periodicities is proposed based on the results reported in (Feder 1993) which can be seen as an implementation of the so called expectation-maximization algorithm (Dempster, Laird, \& Rubin, 1977). In (Spence \& Clarke, 2000), a likelihood computation is put in terms of a least mean square (LMS) residual power expression which is evaluated for different test periods. The estimated period is chosen as the one minimising the LMS residual power. In the field of music and speech analysis the pitch period (fundamental frequency) estimation is very important. For short-time estimation of pitch period, the algorithms that find the average fundamental frequency using autocorrelation or linear prediction techniques are the most commonly used (Guangyu \& Shize, 2009), (Peeters, 2006), (Bernardin, 2006), (Min, Yingchun, \& Zhaohui, 2005).

We propose a period estimation algorithm that takes advantage on the zero mean nature of the signals. This scheme is not restricted to sinusoidal dominant frequency, do not requires a limited frequency band known a priori and is computationally simple. The period information is then used for the solution of velocity/position estimation based only in inertial information with no aiding sensors nor explicit dynamical model. Summarizing, we propose a method that allows to adjust the velocity/position estimations for the case in which the acceleration measurements, which could be biased and contaminated by a zero mean noise, correspond to an oscillating periodical motion with zero mean velocity and position. This proposal compensates the drift using information of the oscillating motion period and can achieve finite-time estimation, for the zero noise and no integration error case.

The rest of the paper is organized as follows, in the next section the problem statement is presented. In section III the possibility of an asymptotic velociy/position estimation is discussed and the idea of using the oscillation period is introduced. Section IV is devoted to the period estimation proposal. In Section V the scheme for finite-time position/velocity estimation is presented. Finally, Section VI presents simulation results.

\section{Problem statement}

Consider a system with the following acceleration dynamics

$$
\ddot{x}(t)=f(x, \dot{x})
$$

and measurement model

$$
\Psi(t)=\ddot{x}(t)+\eta+\beta,
$$

where $\eta$ is a bounded zero-mean noise and $\beta$ is a constant bias. $x \in \mathbb{R}$ and $f(x, \dot{x})$ is an unknown nonlinear function. Mechanical systems are among a wide spectrum of physical systems encompassed by (1). It is assumed that the position $x$, and hence $\dot{x}, \ddot{x}$, have a periodic or quasi-periodic behaviour, more precisely it is considered that position is oscillating between two bounds. There exist two constants such that $B_{m} \leq x^{(i)}(t) \leq B_{M}, \quad \forall t>0, i=0,1,2$.

The objective of this work is to present an alternative solution for velocity and position estimation in the case of only acceleration measurement for a class of periodical signals, i.e., $x^{(i)}(t)=x^{(i)}\left(t+T_{f}\right)$ for some finite $T_{f}$.

\section{Velocity estimation}

\subsection{Velocity estimation through averaging}

In the following, the super index $\langle i\rangle$ is used to indicate the $i$-th time integral of a function $y: R \rightarrow R:$

$$
y^{<i>}(t)=\int_{0}^{t} \ldots \int_{0}^{\tau_{2}} y\left(\tau_{1}\right) d \tau_{1} \ldots d \tau_{i}
$$


While $y^{<i>}\left(t_{0}, t\right)$, with $t \geq t_{0}$, indicates the interval of evaluation:

$$
y^{<i>}\left(t_{0}, t\right)=\int_{t_{0}}^{t} \ldots \int_{t_{0}}^{\tau_{2}} y\left(\tau_{1}\right) d \tau_{1} \ldots d \tau_{i} .
$$

Define

$$
\eta_{\beta}^{<i>}(t)=\eta^{<i>}(t)+\beta^{<i>}(t) .
$$

Now, in order to obtain velocity estimation we proceed to integrate $\Psi(t)$ directly from the measurement 22. Hence,

$$
\begin{aligned}
& \Psi^{<1>}(t)=\dot{x}(t)-\dot{x}(0)+\eta_{\beta}^{<1>}(t) \\
& \Psi^{<2>}(t)=x(t)-x(0)-\dot{x}(0) \cdot t+\eta_{\beta}^{<2>}(t)
\end{aligned}
$$

and dividing (7) by $t$ we obtain

$$
\frac{\Psi^{<2>}(t)}{t}=-\dot{x}(0)+\frac{x(t)-x(0)+\eta_{\beta}^{<2>}(t)}{t} .
$$

Then, define the velocity estimation, $\hat{\dot{x}}(t)$, as follows:

$$
\begin{aligned}
\hat{\dot{x}}(t) & =\Psi^{<1>}(t)-\frac{\Psi^{<2>}(t)}{t} \\
& =\dot{x}(t)+\eta_{\beta}^{<1>}(t)-\frac{x(t)-x(0)+\eta_{\beta}^{<2>}(t)}{t} .
\end{aligned}
$$

Let us recall that the position is bounded. If additionally, it holds that $\forall t \geq 0,\left|\eta_{\beta}^{<i>}(t)\right| \leq \bar{\eta}_{\beta}^{<i>}$ for some constant $0 \leq \bar{\eta}_{\beta}^{<i>}$ (i.e. $\beta=0$ ), the next limit can be obtained

$$
\lim _{t \rightarrow \infty}\left\{\frac{x(t)-x(0)+\bar{\eta}_{\beta}^{<2>}}{t}\right\}=0 .
$$

Therefore, for $t$ "sufficiently" large

$$
\hat{\dot{x}}(t) \simeq \dot{x}(t)+\eta_{\beta}^{<1>}(t)
$$

A similar averaging approach can be applied for position estimation.

Note that the assumption $\beta=0$ is a limitation of the described averaging procedure and it is not required for the method presented in this work. The above estimation is asymptotic without a possibility to regulate the speed of convergence. The amount of time, which is necessary to obtain a reliable estimation, will depend on the magnitude of the bounds of position and the constant bound for the noise integrals (a very strong and even unrealistic assumption when any measurement bias is present). In addition, integration over an infinite time interval can be technically difficult without a significant accumulation of computation and, as a consequence, estimation errors even in the case of the known initial condition $\dot{x}(0)$.

In the present paper a finite time solution is proposed. In fact, a possibility to find a solution in finite time is closely related with period knowledge. This statement is further discussed in the following subsection.

\subsection{Finite time velocity estimation}

Assume that $\ddot{x}, \dot{x}$ and $x$ are unbiased periodic functions with period $T_{f}$ (their integrals over a period are equal to zero). In this paper it is considered that a bias only can be present in the acceleration measurement. In fact, if $\dot{x}$ or $\ddot{x}$ have a bias, then $x$ would not be periodical and bounded. Moreover, 
a constant bias in position cannot be identified from acceleration or velocity measurements because it is not reflected in such measurements. Now, consider the equations (6) and 77 evaluated at $T_{f}$

$$
\begin{aligned}
& \Psi^{<1>}\left(T_{f}\right)=\dot{x}\left(T_{f}\right)-\dot{x}(0)+\eta_{\beta}^{<1>}\left(T_{f}\right) \\
& \Psi^{<2>}\left(T_{f}\right)=x\left(T_{f}\right)-x(0)-\dot{x}(0) \cdot T_{f}+\eta_{\beta}^{<2>}\left(T_{f}\right)
\end{aligned}
$$

where $x\left(T_{f}\right)=x(0)$ and $\dot{x}\left(T_{f}\right)=\dot{x}(0)$. Hence, an estimation $\hat{\dot{x}}(0)$ of $\dot{x}(0)$ can be obtained at $t=T_{f}$ :

$$
\begin{aligned}
\hat{\dot{x}}(0) & =-\frac{\Psi^{<2>}\left(T_{f}\right)}{T_{f}} \\
& =\dot{x}(0)-\frac{\eta_{\beta}^{<2>}\left(T_{f}\right)}{T_{f}} .
\end{aligned}
$$

Then, if period is known, an estimation of velocity can be derived using equations (12) and (15):

$$
\hat{\dot{x}}(t)=\Psi^{<1>}(t)+\hat{\dot{x}}(0) \text {. }
$$

Next, the same approach can be repeated for position estimation. Consider the following expression for

$$
\Psi^{<3>}\left(T_{f}\right)=x^{<1>}\left(T_{f}\right)-x^{<1>}(0)-\dot{x}(0) \cdot T_{f}-\frac{1}{2} \hat{\dot{x}}(0) \cdot T_{f}^{2}+\eta_{\beta}^{<3>}\left(T_{f}\right),
$$

thereby, an estimation of the position initial condition can be obtained as

$$
\hat{x}(0)=-\frac{\Psi^{<3>}\left(T_{f}\right)}{T_{f}}+\frac{1}{2} \hat{\dot{x}}(0) T_{f}
$$

Remark 1: Note that the estimation would be exact in the absence of noise (a constant measurement bias can be identified and compensated provided the period is available). However, in the presence of noise, a finite-time estimation whose accuracy will depend on the noise magnitude, assumed to be bounded, can be achieved by using the value of the period.

\subsubsection{Importance of period knowledge}

Note that $x(t)=x(0)$ may hold for different time instants, but the correct estimation of velocity can only be ensured for $t=\kappa T_{f}$ with $\kappa \in \mathbb{N}$. It implies that estimation of the initial condition, $\dot{x}(0)$, without knowledge of the period may lead to big errors. To illustrate this idea, consider the next expression for acceleration

$$
\ddot{x}(t)=\sin (1.5 t)+\sin (4.2 t)
$$

which is plotted with its corresponding velocity and position signals in Fig. 1. Suppose that measurements starts at $t=A$. Note that there is a subperiod, between times $A$ and $B$, for both acceleration and velocity. Given that only the acceleration is available, this zero mean subperiod can be wrongly considered as the real period causing the error term $(x(B)-x(A)) /(B-A)$ to be present in the estimation $\hat{\dot{x}}(0)$, see Eq. 15 . A solution to the problem of period estimation is presented in the following Section 4 .

\section{Period Identification}

The algorithm for period estimation proposed in this paper is based on the features of the phase portrait $(\dot{x}, \ddot{x})$. To introduce the idea, consider the phase portrait $(\dot{x}, \ddot{x})$ of $(17)$ depicted in Fig. 2 It is clear that after one period the trajectories pass through each point at least once. Nevertheless, there are a number of points and furthermore trajectory segments, where the trajectory passes more than once over a cycle. If we can select a point which is reached only once over a whole period, then an estimation of the period can be obtained by detecting the time elapsed between two consecutive 

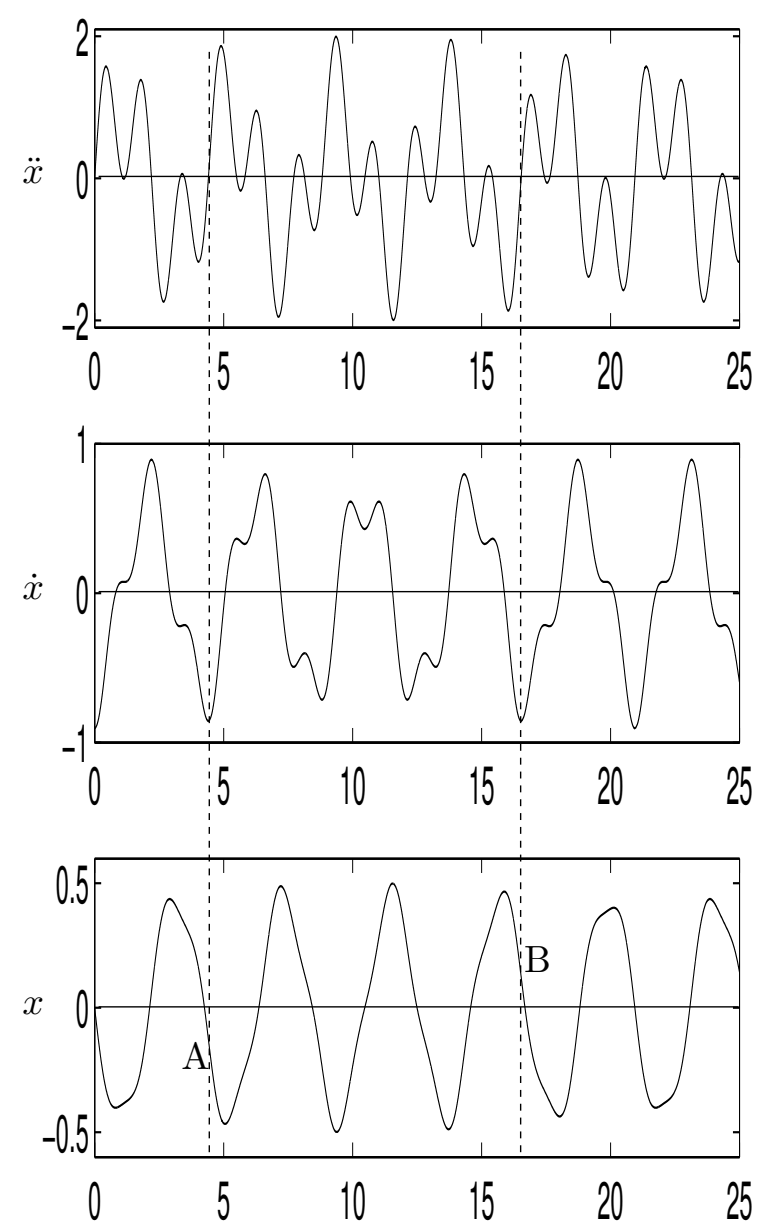

Figure 1. Acceleration, Velocity and Position versus time (sec).

passings over such point. The question is how to properly select this point.

Recall that the real phase portrait is not available because there is no velocity measurement. From Equation (6) it can be seen that the velocity estimation, $\dot{x}$, is shifted by its initial condition. However, it is possible to obtain a period estimation under the next assumptions:

S1. $\dot{x}$ and $\ddot{x}$ are zero-mean periodical signals with unknown period $T_{f}$.

S2. On the period $T_{f}, \dot{x}$ has a unique global extremum, either a maximum or a minimum, represented by $\dot{x}_{M}$.

Remark 2: Note that, as a consequence of the bounded nature of noise and bias, the time integrals $\eta_{\beta}^{<i>}(t), i=$ 1,2 are bounded $\forall t \in\left[0, T_{f}\right]$.

Remark 3: The Assumption S2 restricts the function $\dot{x}$ to have only a single occurrence of the respective global extremum over one period. In (17), for instance, the maximum for $\dot{x}$ does not fulfills S2.

In (Spence \& Clarke, 2000) an equivalent of assumption S2 is also considered while S1 is not required. However, the period estimation uses optimization of a criterion which involves either residual or signal power over a set of data using a set of trial periods, as they mention the basis of its time domain estimator is 'trial and error' using a number of test periods. While not requiring S1 or S2, methods like those in (Peeters, 2006) and (Guangyu \& Shize, 2009) are devoted to identify a sinusoidal dominant frequency and the results in (Veluvolu \& Ang, 2011) assumes band limited signals consisting of multiple sinusoidal components. The scheme in the present work do not requires that sinusoidal composition paradigm. In this paper it is proposed the detection of two consecutive passings trough an extremum $\dot{x}_{M}$ which comply with S2. The successful $\dot{x}_{M}$ detection involves 


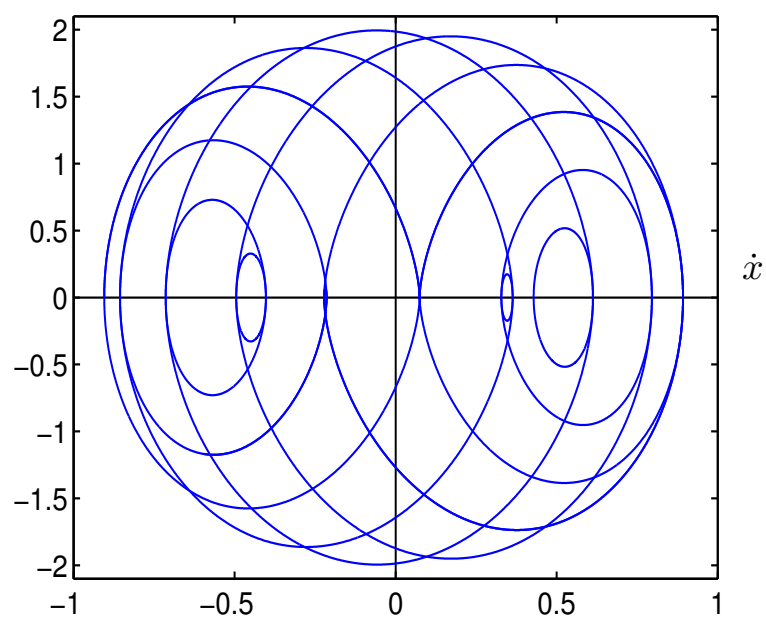

Figure 2. Phase portrait $(\dot{x}, \ddot{x})$ for 17 .

estimation of the drift with respect to the integration time elapsed. This drift estimation is addressed next.

\subsection{Estimation of an upper bound for $\eta_{\beta}^{<1>}$}

In general, acceleration is a noisy measurement. Additionally, the accuracy of integration is limited by sampling time. The aforementioned factors lead to integration drift. Obviously, such a drift is worsen by the presence of measurement bias. In (Estrada, Efimov, \& Perruquetti 2014), the problem of position and velocity estimation was addressed assuming unbiased acceleration measurements and knowledge of a bound for the integration error. In the present proposal the assumptions in (Estrada, Efimov, \& Perruquetti, 2014) are relaxed allowing the presence of biased measurements and dropping the requirement of giving an a priori bound for the integration drift. That is achieved thanks to the introduction of a time varying estimation of the integration drift, based on the more realistic assumptions of having bounds for the noise and bias magnitudes. The expressions used for obtaining such an estimation are presented here. Further details regarding derivation of those expressions are included in the Appendix.

Define $\bar{\eta}^{<i>}\left(t_{0}, t\right)$ and $\bar{\beta}^{<i>}\left(t_{0}, t\right)$ as upper bounds for $\eta^{<i>}\left(t_{0}, t\right)$ and $\beta^{<i>}\left(t_{0}, t\right)$, on the interval $t \in\left[t_{0}, t\right]$, respectively $(i=0,1,2)$. Recall that $\bar{\eta}^{<0>}$ and $\bar{\beta}^{<0>}$, which do not depend on $t$, are assumed to be known. The bound of the drift due to the contribution of the zero mean noise integration, over a finite interval $\left[t_{0}, t\right], t_{0} \leq t$, is computed as follows

$$
\bar{\eta}^{<1>}\left(t_{0}, t\right)=4 \bar{\eta}^{<0>} \frac{\sqrt{t-t_{0}+f_{s}^{-1}}}{\sqrt{f_{s}}},
$$

where $f_{s}$ is the sampling frequency, $t_{0}$ is the initial time of integration. Expression $\sqrt{18}$ is an estimation of the drift due to integration of noise, see the Appendix section for details. The upper bound for drift due to bias integration is obtained straightforward by integrating its constant upper bound. Therefore,

$$
\bar{\beta}^{<1>}\left(t_{0}, t\right)=\bar{\beta}^{<0>} \cdot\left(t-t_{0}\right) .
$$

Finally, the upper bound of the combined drift is

$$
\bar{\eta}_{\beta}^{<1>}\left(t_{0}, t\right)=\bar{\eta}^{<1>}\left(t_{0}, t\right)+\bar{\beta}^{<1>}\left(t_{0}, t\right)
$$




\subsection{Period Identification Algorithm}

Recall that $\dot{x}_{M}$ denotes the global extremum in velocity. The algorithm for period estimation should perform the following tasks.

(1) To ensure the detection, once each period, of the extremum $\dot{x}_{M}$.

- Using the $\bar{\eta}_{\beta}^{<1>}\left(t_{0}, t\right)$ bound, discriminate $\dot{x}_{M}$ from any other local extremum.

- In addition to possible extrema outside of the $\bar{\eta}_{\beta}^{<1>}\left(t_{0}, t\right)$-vicinity, there may be local ones, not present on the real $\dot{x}$ but induced by the integration of noise, inside such a vicinity.

- The above implies that it is necessary to wait until the estimated $\dot{x}$ exits some vicinity of the last $\dot{x}_{M}$ candidate in order to decide that this candidate can be considered as a new occurrence of $\dot{x}_{M}$.

(2) The integral of acceleration, on which $\dot{x}$ estimation and $\dot{x}_{M}$ detection is based, should be restarted once each period in order to tackle drift accumulation.

- This reset can be performed after a new $\dot{x}_{M}$ occurrence has been confirmed.

If the above conditions are fulfilled then an estimation of the period can be made as the difference between two consecutive detected occurrences of $\dot{x}_{M}$. Prior to present the algorithm some definitions are introduced:

- $\alpha$ - is a parameter to be selected as 1 or -1 in order to detect a global maximum, or a global minimum respectively.

- $\dot{x}_{M-}$ - is the extremum, different from $\dot{x}_{M}$, whose amplitude is the closest to $\dot{x}_{M}$.

- $\Psi^{<1>}\left(t_{a}, t_{b}\right)$ - indicates the integral of $\Psi$ from $t=t_{a}$ to $t=t_{b}$.

- $\delta(t)$ - is a time varying parameter upper bounding the drift due to noise and bias integration.

Next we present the proposed period detection algorithm:

Algorithm A1.

Initialization of variables

$$
\begin{aligned}
& \alpha= \pm 1, t_{0}=0, t_{1}=0, f l=0 \\
& \delta(t)=\bar{\eta}_{\beta}^{<1>}\left(t_{0}, t\right) \\
& \Psi_{M}^{<1>}=0
\end{aligned}
$$

C0. If $\alpha \Psi^{<1>}\left(t_{0}, t\right)>\alpha \Psi_{M}^{<1>}-\delta(t) f l \underline{\text { then }}$

$$
\begin{aligned}
\Psi_{M}^{<1>} & =\Psi^{<1>}\left(t_{0}, t\right), \\
t_{1} & =t, \\
f l & =0 .
\end{aligned}
$$

C1. If $\alpha \Psi^{<1>}\left(t_{1}, t\right)<-2 \delta(t)$ and $f l=0$ then

$$
\begin{aligned}
\Psi_{M}^{<1>} & =0, \\
\hat{T}_{f} & =t_{1}-t_{0}, \\
t_{0} & =t_{1}, \\
f l & =1,
\end{aligned}
$$

Remark 4: $\delta$ is evaluated according to 20 and updated every sampling time.

We state the next claims:

Lemma 1: Each occurrence of $\dot{x}\left(T_{M}^{i}\right)=\dot{x}_{M}$ is followed by only one fulfilment of condition $\mathbf{C 1}$ with a value for $t_{1}$ such that

$$
\dot{x}\left(t_{1}\right) \in\left[\dot{x}_{M}-\bar{\eta}_{\beta}^{<1>}\left(\left|T_{M}^{i}-t_{1}\right|\right), \dot{x}_{M}\right] .
$$

Proposition 1: If assumptions S1-S2 are fulfilled then, an estimation of the real period, $T_{f}$, can be obtained by 
means of the algorithm A1, with $\delta(t)=\bar{\eta}_{\beta}^{<1>}\left(t_{0}, t\right)$, provided that

$$
\frac{1}{2}\left|\dot{x}_{M}-\dot{x}_{M-}\right|>\bar{\eta}_{\beta}^{<1>}\left(T_{f}\right)
$$

Assume that $\alpha=1$ is selected. Then, the algorithm A1 proceeds in the following manner:

- The condition $\mathbf{C 0}$ at the beginning is $\alpha \Psi^{<1>}(0, t)>0$, then it is fulfilled for any positive quantity of the integral $\alpha \Psi^{<1>}(0, t)$.

- After the first fulfillment of $\mathbf{C 0}$ the assigment $\Psi_{M}^{<1>}=\Psi^{<1>}(0, t)$ is done in order to save the new maximum and the time of its occurrence is saved on $t_{1}$.

- The assignment $t_{1}=t$, at each new maximum, implies that the integral $\Psi^{<1>}\left(t_{1}, t\right)$ is restarted.

- Due to $f l=1$ assignment inside $\mathbf{C 1}$, once the latter condition is verified it will not be triggered again before a new $\mathbf{C O}$ verification.

- It can be seen that the condition $\mathbf{C 1}$ contains the criteria which decides when a detected maximum is considered as an occurrence of the global maximum $\dot{x}_{M}$. In fact, before the completion of one period, it is possible that a local maximum is considered as $\dot{x}_{M}$. Nevertheless, as it is shown in the proof of Lemma 1 . this is anymore the case after the first occurrence of $\dot{x}_{M}$.

- Finally, note that when condition $\mathbf{C 1}$ is fulfilled, the integral $\Psi^{<1>}\left(t_{0}, t\right)$ contains the integrated acceleration with an initial time corresponding to the last maximum. Additionally, the condition $\mathbf{C O}$ takes the form $\Psi^{<1>}\left(t_{0}, t\right)>-\delta(t)$. This change in the form of condition $\mathbf{C 0}$ is needed in order to detect the occurrence of next maximum taking into account a new drift $\eta_{\beta}^{<1>}\left(t_{0}, t\right)<\delta(t)$.

\subsection{Period estimation simulations}

The algorithm A1 was applied to the identification of the period, $T_{f}=20.944$, of $(17)$ contaminated only by a zero mean noise, $\eta$, fulfilling that $|\eta| \leq 0.024$. The integration step, the simulation time for producing the signals was fixed to $1 \times 10^{-4} \mathrm{~s}$, while the sampling period, the time at which measurements are made, was fixed to $6 \times 10^{-3} \mathrm{~s}$. The parameters $\eta^{<0>}=0.024$ and $\beta^{<0>}=0$ are used for $\delta(t)$ evaluation. $\alpha=-1$ for a global minimum detection was chosen. Indeed, the maximum of this signal appears twice over a period, failing to fulfill Assumption S2. The phase portrait of the signals $\left(\Psi^{<1>}, \Psi\right)$ is depicted in Fig. 3 whereas in Fig. 4 the period estimation is plotted.

$\Psi$

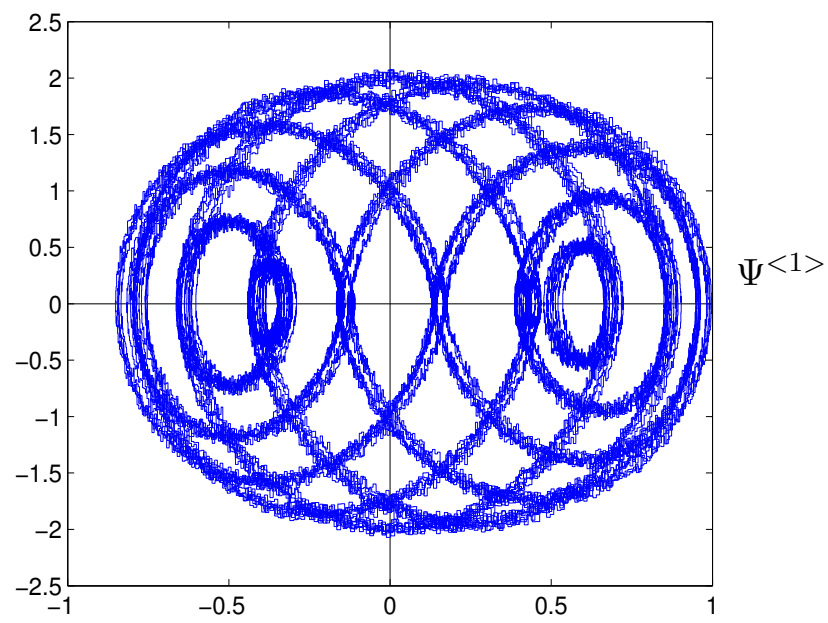

Figure 3. Phase portrait $\left(\Psi^{<1>}, \Psi\right)$ for noisy measurements $(|\eta|<0.024)$ of 17 . 


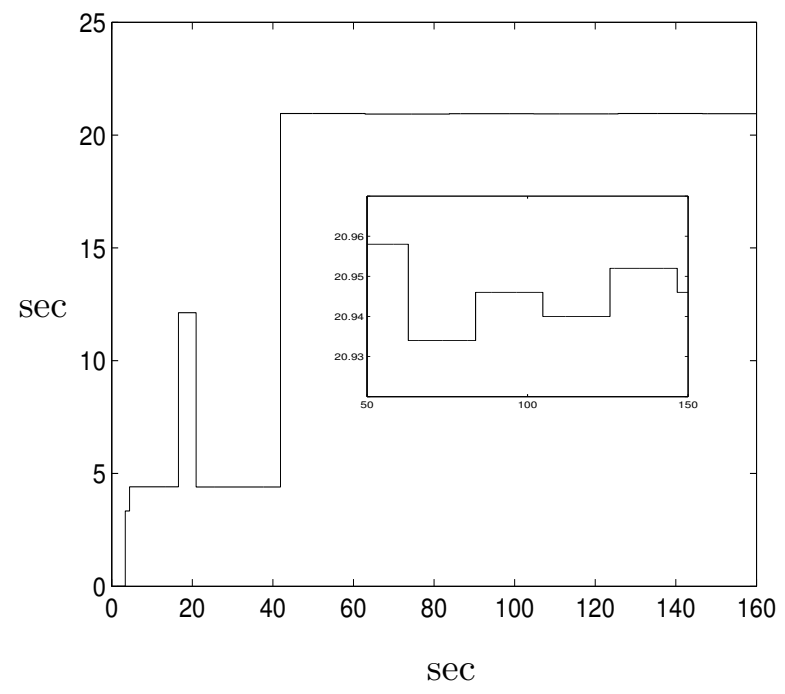

Figure 4. Estimated period $\hat{T}_{f}$ for signal 17 with noise.

\section{Velocity and position estimation based on period estimation}

The period estimation, $\hat{T}_{f}$, obtained in the previous section can be used to estimate $\dot{x}_{M}$ and in turn to obtain a real time velocity estimation. The fact that $\dot{x}_{M}$ is estimated at each period will be used in order to compensate the drift due to errors previously mentioned. Define $\hat{T}_{f}^{i}$ as the period estimation corresponding to the time $T_{M}^{i}$, namely the $i-t h$ occurrence of $\dot{x}_{M}$. Define $\hat{T}_{M}^{(i-1)}$ and $\hat{T}_{M}^{(i)}$ as the corresponding extremum occurrence estimated time. Such estimation will correspond to the ones obtained at each fulfillment of the condition $\mathbf{C 1}$ of algorithm A2, i.e. $\hat{T}_{M}^{(i)}=t_{1}$ and $\hat{T}_{M}^{i-1}=t_{0}$. Thus, based on (14) we obtain

$$
\hat{\dot{x}}\left(\hat{T}_{M}^{i-1}\right)=-\frac{\Psi^{<2>}\left(\hat{T}_{M}^{i-1}, \hat{T}_{M}^{i}\right)}{\left(\hat{T}_{f}^{i}\right)} .
$$

Analogously, an estimation $\hat{x}\left(T_{M}^{i}\right)$ can be obtained by using the estimated period $\hat{T}_{f}^{i}$ and the third integral of $\Psi$ that is

$$
\begin{aligned}
\Psi^{<3>}\left(T_{M}^{i-1}, T_{M}^{i}\right)= & x^{<1>}\left(T_{M}^{i}\right)-x^{<1>}\left(T_{M}^{i-1}\right) \\
& -x\left(T_{M}^{i-1}\right)\left(T_{M}^{i}-T_{M}^{i-1}\right) \\
& -\frac{1}{2} \dot{x}\left(T_{M}^{i-1}\right)\left(T_{M}^{i}-T_{M}^{i-1}\right)^{2} \\
& +\eta^{<3>}\left(T_{M}^{i-1}, T_{M}^{i}\right),
\end{aligned}
$$

then, assuming that $x^{<1>}\left(T_{M}^{i}\right)=x^{<1>}\left(T_{M}^{i-1}\right)$, the next estimation can be obtained

$$
\hat{x}\left(\hat{T}_{M}^{i}\right)=-\frac{\Psi^{<3>}\left(\hat{T}_{M}^{i-1}, \hat{T}_{M}^{i}\right)}{\hat{T}_{f}}+\frac{1}{2} \hat{\dot{x}}\left(\hat{T}_{M}^{i-1}\right) \hat{T}_{f}
$$

In the ideal case of zero noise, no bias, exact integration and exact detection of the extremum instants $T_{M}^{i}$ 's, we have that $\Psi^{<1>}\left(\hat{T}_{M}^{i-1}, \hat{T}_{M}^{i}\right)=0(\delta(t)=0)$. Although, in general $\Psi^{<1>}\left(\hat{T}_{M}^{i-1}, \hat{T}_{M}^{i}\right) \neq 0$, the assumption $\dot{x}\left(\hat{T}_{M}^{i-1}\right)=\dot{x}\left(\hat{T}_{M}^{i}\right)$ is made in order to obtain the period estimation $\hat{T}_{f}^{i}$. According to the aforementioned assumption an estimation of the error $\eta_{\beta}^{<1>}$ can be 
obtained as

$$
\hat{\eta}^{<1>}\left(\hat{T}_{M}^{i}\right)=\Psi^{<1>}\left(\hat{T}_{M}^{i-1}, \hat{T}_{M}^{i}\right)
$$

Using (28) we can obtain an estimation of the errors $\eta^{<2>}$ and $\eta^{<3>}$ allowing to compensate them in $\Psi^{<2>}$ and $\Psi^{<3>}$, before computing $\hat{\dot{x}}\left(\hat{T}_{M}^{(i)}\right)$ and $\hat{x}\left(\hat{T}_{M}^{(i)}\right)$ respectively. In order to do that, we consider $\hat{\eta}^{<1>}\left(T_{M}^{i}\right)$ as the result of the integration of the constant magnitude $\frac{\hat{\eta}^{<1>}\left(\hat{T}_{M}^{i}\right)}{\hat{T}_{f}^{i}}$ over the interval $\left[\hat{T}_{M}^{i-1}, \hat{T}_{M}^{i}\right]$. Thereby, the next error estimations are obtained

$$
\begin{aligned}
\hat{\eta}^{<2>}\left(\hat{T}_{M}^{i}\right) & =\frac{1}{2} \Psi^{<1>}\left(\hat{T}_{M}^{i-1}, \hat{T}_{M}^{i}\right) \hat{T}_{f} \\
\hat{\eta}^{<3>}\left(\hat{T}_{M}^{i}\right) & =\frac{1}{6} \Psi^{<1>}\left(\hat{T}_{M}^{i-1}, \hat{T}_{M}^{i}\right) \hat{T}_{f}^{2} .
\end{aligned}
$$

Compensating (25) and 27) with the above errors yields

$$
\begin{aligned}
\hat{\dot{x}}\left(\hat{T}_{M}^{i}\right)= & -\frac{\Psi^{<2>}\left(\hat{T}_{M}^{i-1}, \hat{T}_{M}^{i}\right)}{\left(\hat{T}_{f}^{i}\right)}+\frac{1}{2} \Psi^{<1>}\left(\hat{T}_{M}^{i-1}, \hat{T}_{M}^{i}\right), \\
\hat{x}\left(\hat{T}_{M}^{i}\right)= & -\frac{\Psi^{<3>}\left(\hat{T}_{M}^{i-1}, \hat{T}_{M}^{i}\right)}{\hat{T}_{f}} \\
& -\left[\frac{1}{2} \hat{\dot{x}}\left(\hat{T}_{M}^{i-1}\right)-\frac{1}{6} \Psi^{<1>}\left(\hat{T}_{M}^{i-1}, \hat{T}_{M}^{i}\right)\right] \hat{T}_{f} .
\end{aligned}
$$

Finally, the estimation of velocity and position is obtained through the next expressions

$$
\begin{aligned}
& \hat{\dot{x}}(t)=\Psi^{<1>}\left(\hat{T}_{M}^{i}, t\right)+\hat{\dot{x}}\left(\hat{T}_{M}^{i}\right), \\
& \hat{x}(t)=\Psi^{<2>}\left(\hat{T}_{M}^{i}, t\right)+\hat{x}\left(\hat{T}_{M}^{i}\right),
\end{aligned}
$$

using (31) and 32 for the evaluation of $\hat{\dot{x}}\left(\hat{T}_{M}^{i}\right)$ and $\hat{x}\left(\hat{T}_{M}^{i}\right)$ respectively.

Remark 5: While the assumptions S1 and S2, altogether with condition 24, restrict the signals and the signal to noise ratio that the estimation algorithm can manage, it is worth mentioning that no a priori bandwidth is assumed, no sinusoidal dominant frequency is required, noise and constant bias are allowed to be present and knowledge of the system model is prescinded.

\section{Example}

The velocity and position scheme presented in previous section was tested through simulations, using the estimations obtained with the algorithm A1. The following Van der Pol oscillator was used for generation of the position, velocity and acceleration signals

$$
\begin{aligned}
& \ddot{x}+0.1(x+1)(x-1)(x-2.2) \dot{x}+x=0 \\
& \Psi(t)=\ddot{x}(t)+\eta(t)+\beta
\end{aligned}
$$

The parameters in (35) where proposed in (Karreman \& Prood, 1995) as a mathematical model for an isometric contraction of the mammalian cardiac muscle. The acceleration signal was contaminated in simulation by a bounded zero mean noise, with $|\eta(t)| \leq 0.11$, and a constant bias, $\beta=0.05$, hence, $\bar{\eta}^{<0>}=0.11$ and $\bar{\beta}^{<0>}$ are used for simulations. The phase portrait $(\ddot{x}, \dot{x})$ of $(35)$, with initial conditions $x_{0}=3$ and $\dot{x}_{0}=-2$, is displayed in Fig. 5 . The estimation results are shown in Fig. 6 and Fig. 7 .

\section{Conclusions}

For a class of oscillatory motion, a scheme for velocity and position estimation using the information of noisy and biased acceleration measurements was presented. The inherent error accumulation, due 


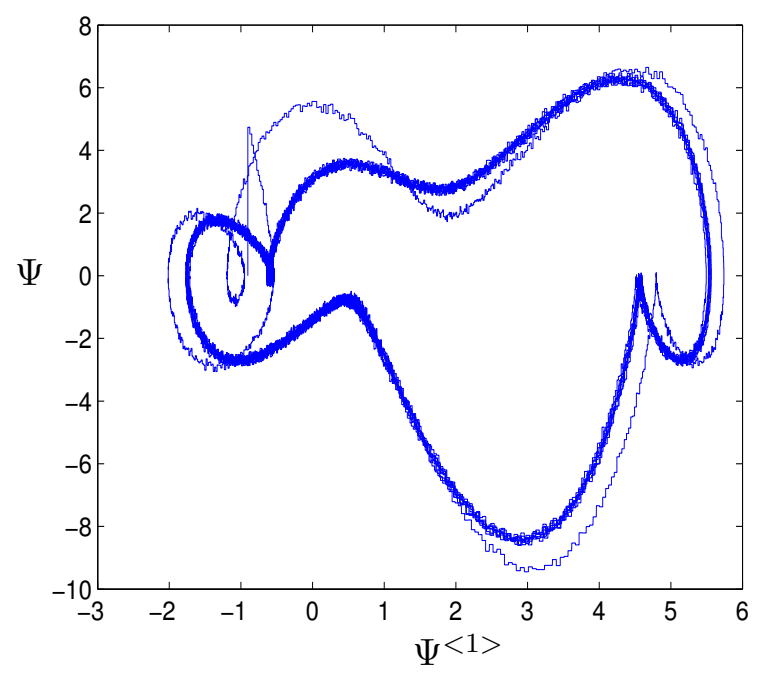

Figure 5. Phase portrait $\left(\Psi^{<1>}, \Psi\right)$ for system $35-36$.

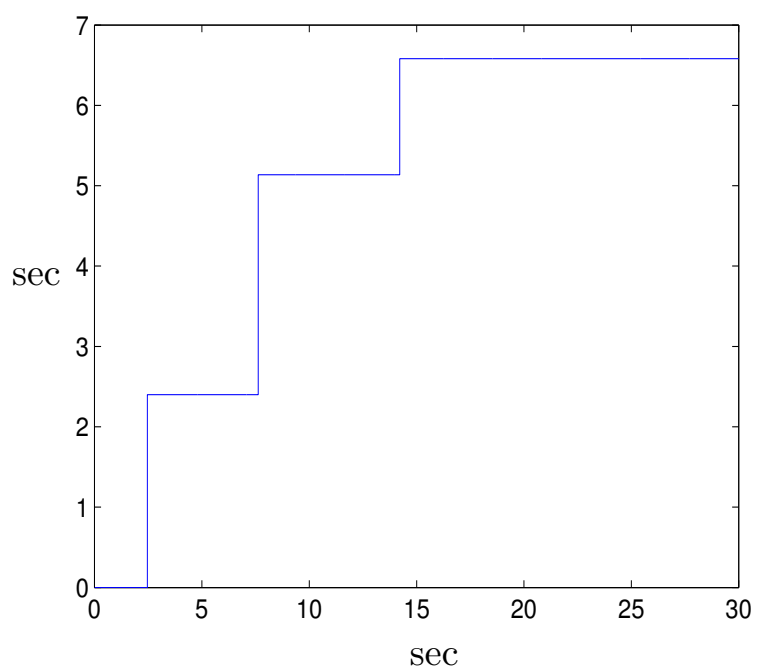

Figure 6. Period estimation for 35- 36 .

to integration drift, is compensated using the value corresponding to the period of oscillation. An algorithm for estimation of the aforementioned period of oscillation is proposed. No model of the motion nor a priori information on the period of the signals is required. As a result, the proposed scheme is suitable as a standalone solution for estimation of position and velocity using only inertial information without any aiding measurements. Further research is directed to the application of the proposed solution on a practical scenario with real measurement data.

\section{Appendix}

Proofs.

Consider that the unique absolute extremum for $\dot{x}$ is a global maximum denoted as $\dot{x}_{M}$ (the proof in the case of a global minimum follows the same rationale). For this case $\alpha=1$ is selected and the 

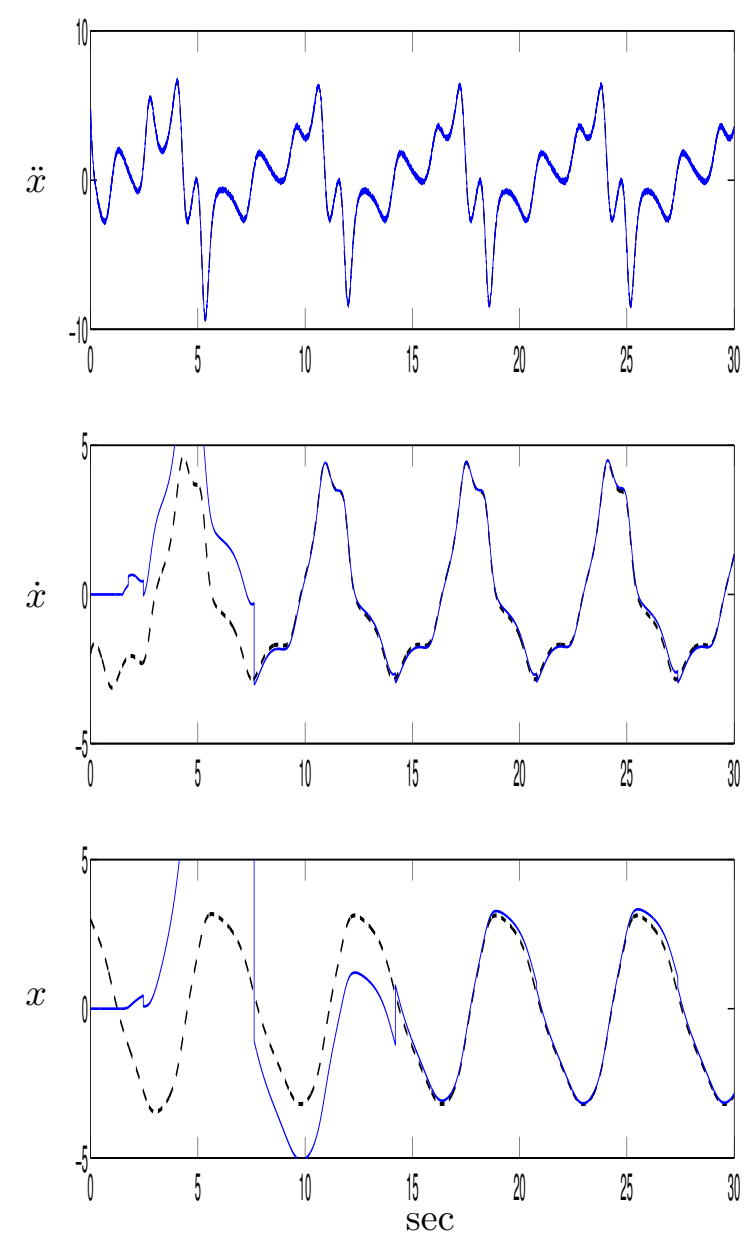

Figure 7. Velocity and position estimation for 35- 36 .

corresponding forms of conditions $\mathbf{C 0}$ and $\mathbf{C 1}$ are rewritten as

$$
\begin{aligned}
& \Psi^{<1>}\left(t_{0}, t\right)>\Psi_{m}^{<1>} \\
& \Psi^{<1>}\left(t_{0}, t\right)>-\delta(t)
\end{aligned}
$$

and

$$
\left.\begin{array}{ccc}
\Psi^{<1>}\left(t_{1}, t\right) & < & -2 \delta(t) \\
f l & = & 0
\end{array}\right\}
$$

Note that condition C0 can take two forms, $37 \mathrm{a}$ for $f l=0$ and $37 \mathrm{~b}$ for $f l=1$.

Proof of Lemma 1. This proof is divided into two parts:

- Localization of the global maximum. This part is focused on the fact that the first fulfillment of Lemma 1

$$
\dot{x}\left(t_{1}\right) \in\left[\dot{x}_{M}-\bar{\eta}_{\beta}^{<1>}\left(\left|T_{M}-t_{1}\right|\right), \dot{x}_{M}\right]
$$

is not affected for the initial conditions $\ddot{x}_{\circ}=\ddot{x}(0), \dot{x}_{\circ}=\dot{x}(0)$ and $x_{\circ}=x(0)$.

Define $T_{M}^{1}$ as the time of first appearance of $\dot{x}_{M}$, i.e., $\dot{x}\left(T_{M}^{1}\right)=\dot{x}_{M}$. Suppose that the initial condition $\dot{x}_{\circ}$ 
is such that

$$
\dot{x}_{M}-\dot{x}_{\circ}>\bar{\eta}_{\beta}\left(0, T_{M}^{1}\right) \text {. }
$$

Then, considering (40), if $\mathbf{C 1}$, i.e. [38), is not fulfilled between $t=0$ and $t=T_{M}^{1}$, implying $\dot{x}$ is not decreasing, we have

$$
\begin{aligned}
\Psi^{<1>}\left(t_{0}, T_{M}^{1}\right) & =\Psi^{<1>}\left(0, T_{M}^{1}\right) \\
& =\dot{x}_{M}-\dot{x}_{\circ}+\eta_{\beta}\left(0, T_{M}^{1}\right) \\
& >\bar{\eta}_{\beta}\left(0, T_{M}^{1}\right)-\left|\eta_{\beta}\left(0, T_{M}^{1}\right)\right| \\
& >0 .
\end{aligned}
$$

Thereby, C0, that is (37a), will be necessarily true for some $t \in\left[t_{0}, T_{M}^{1}\right]$. This implies that the time $t_{1}$ captured due to $\mathbf{C 0}$ verifies $(39)$.

On the other hand, suppose that $\mathbf{C 1}$, see (38), is true before $T_{M}^{1}$, at a time which will be denoted as $T_{C 1}^{1}$. This implies that $\Psi^{<1>}\left(t_{0}, T_{C 1}^{1}\right)$ has decreased more than $\bar{\eta}_{\beta}^{<1>}\left(t_{0}, T_{C 1}^{1}\right)$, because $\eta_{\beta}\left(t_{0}, T_{C 1}^{1}\right)<\delta(t)=$ $\bar{\eta}_{\beta}^{<1>}\left(t_{0}, T_{C 1}^{1}\right)$. The assignments $\Psi_{M}^{<1>}=0$ and $f l=1$ due to $\mathbf{C 1}$ implies that condition $\mathbf{C 0}$ takes the form (37b). Now, note that the latter must be fulfilled before a new true event of $\mathbf{C 1}$ : Since $t_{0}$ is reseted to the time of occurrence of the last largest value of $\Psi^{<1>}\left(t_{0}, T_{C 1}^{1}\right)$, one obtains that

$$
\Psi^{<1>}\left(t_{0}, T_{C 1}^{1}\right)<-2 \delta\left(t_{0}, T_{C 1}^{1}\right)
$$

holds, after the assignments in $\mathbf{C 1}$, with $x_{M}$ still to appear. Then, there exist a time $t^{*}$ such that

$$
\dot{x}\left(t^{*}\right)=\dot{x}\left(t_{0}\right) ; \quad \text { with } t^{*}<T_{M}^{1}<T_{f},
$$

and for $t=t^{*}$ one obtains

$$
\begin{aligned}
\Psi^{<1>}\left(t_{0}, t^{*}\right) & =\dot{x}\left(t^{*}\right)-\dot{x}\left(t_{0}\right)+\eta_{\beta}^{<1>}\left(t_{0}, t^{*}\right) \\
& \geq-\left|\eta_{\beta}^{<1>}\left(t_{0}, t^{*}\right)\right|>-\delta(t) .
\end{aligned}
$$

Then, (37b is fulfilled and $\mathbf{C 0}$ takes the form $(37 \mathrm{a})$ again.

From the above, it is concluded that condition (37) is verified, independently of the initial conditions, for some time $t_{1}$ such that $(39)$ holds.

- Successive global maximum detections. Once $t_{1}$ satisfies $(39)$, condition $\mathbf{C 1}$ is fulfilled only once after each $\dot{x}_{M}$ occurence. This ensures that no local extremum is mistaken as the global one. Consider that C1 is true after 39 is satisfied then,

$$
\dot{x}\left(t_{1}\right) \in\left[\dot{x}_{M}-\bar{\eta}_{\beta}^{<1>}\left(\left|T_{M}-t_{1}\right|\right), \dot{x}_{M}\right]
$$

and note that $\mathbf{C 0}$ must be true before $\mathbf{C 1}$ can be satisfied again. Recall that the local extremum closest in magnitude to $\dot{x}_{M}$ is $\dot{x}_{M}^{-}$. Then, for $\dot{x}(t)=\dot{x}_{M}^{-}$, after 39 holds, we obtain

$$
\begin{aligned}
& \Psi^{<1>}\left(t_{0}, t\right)=\dot{x}_{M}^{-}-\dot{x}\left(t_{0}\right)+\eta_{\beta}^{<1>}\left(t_{0}, t\right) \\
& \Psi^{<1>}\left(t_{0}, t\right) \leq \dot{x}_{M}^{-}-\dot{x}_{M}+\eta_{\beta}^{<1>}\left(t_{0}, t\right)+\bar{\eta}_{\beta}^{<1>}(T f) .
\end{aligned}
$$

From 24 we have that $\dot{x}_{M}^{-}-\dot{x}_{M}<-2 \delta\left(T_{f}\right)$, hence

$$
\begin{aligned}
& \Psi^{<1>}\left(t_{0}, t\right)<-2 \delta\left(T_{f}\right)+\delta\left(T_{f}\right) \\
& \Psi^{<1>}\left(t_{0}, t\right)<-\delta\left(T_{f}\right)
\end{aligned}
$$

thus, $\dot{x}(t)$ must be larger than $\dot{x}_{M}^{-}$in order to fulfill again C0. Thus, after the first time [39] is fulfilled, Co cannot be triggered due to a local extremum in the original $\dot{x}$.

This concludes the proof of Lemma 1

Proof of Proposition 1. Proposition 1 is a straightforward result of Lemma 1] Note that after (39) holds, for each subsequent $\mathbf{C 1}$ verification, the variables $t_{0}$ and $t_{1}$ will contain the time of 
detection of two consecutive global maxima of $\Psi^{<1>}$. The condition $(39)$ cannot be ensured before a whole period has elapsed, then at least two periods are needed in order to ensure that a correct estimation of $T_{f}$ is obtained. As a consequence, the first period estimation may occur during the third period after launching the algorithm (C1 verification after two periods will occur on the third one).

\section{Time Varying $\bar{\eta}^{<1>}$}

We mentioned before that integration of noise leads to a drift. The magnitude of this drift increases with the time of integration, on the contrary to the mean value of the noise measurements, that approaches zero as time increases due to the zero mean assumption. In (Thong et al., 2002) it is validated that, for the sampled case, the value of the integral of zero mean noise, with uncorrelated measurements, is a random value with Gaussian distribution. The variance of such Gaussian distribution increases with time. In fact, the selection of a constant $\delta$, in Algorithm A1, implies in some sense knowledge of both the bound of noise and some upper bound for the period.

For the sake of completeness a brief description of the procedure in (Thong et al. 2002) to obtain the expression for the variance of the drift due to integration of noise at a time $T$ is included next. Consider an arbitrary time interval $T$, and the $N$ values of the noise obtained over this interval as a result of sampling at frequency $f_{s}$, i.e. $N=T f_{s}$. Define $\eta[i]$ as the sampled noise values. The average of the noise values over the $N$ sample points is:

$$
D_{N}=\frac{1}{N} \sum_{i=1}^{N} \eta[i] .
$$

$D_{N}$ approaches zero as $N \rightarrow \infty$, but for finite $N$ will in general be nonzero. The square of the mean of $D_{N}$ is given by

$$
\begin{aligned}
E\left[\left(D_{N}\right)^{2}\right]= & \frac{1}{N^{2}} E[(\eta[1]+\ldots+\eta[N]) \times \\
& (\eta[1]+\ldots+\eta[N])]
\end{aligned}
$$

where $E\left[\left(D_{N}\right)^{2}\right]$ denotes the expected value. Expanding the brackets, using the symmetry condition $E[\eta[i] \cdot \eta[j]]=E[\eta[j] \cdot \eta[i]]$ and using the $p$-th lag of the autocorrelation function, under the assumption of stationary data, defined as

$$
r[p]=E[\eta[i] \cdot \eta[i+p]]
$$

the next expression is obtained

$$
\begin{aligned}
E\left[\left(D_{N}\right)^{2}\right] & =R_{1}+R_{2} \\
\text { where } \quad R_{1} & =\frac{r[0]}{N} \\
R_{2} & =\frac{2}{N^{2}} \sum_{j=1}^{N-1} r[j](N-j) .
\end{aligned}
$$

The term $R_{1}$ is the contribution of noise samples in the absence of correlations between samples and $R_{2}$ is the respective contribution from correlations. For the white noise model no correlation between samples is assumed, that is $R_{2}=0$. Let $\sigma_{\eta}$ be the standard deviation of the noise. Using $R_{2}=0$ and $r[0]=\sigma_{\eta}^{2}$ in equation 44 one gets

$$
E\left[\left(D_{N}\right)^{2}\right]=\frac{\sigma_{\eta}^{2}}{N} .
$$


Taking square roots on both sides of the above equation yields (Thong et al. 2002)

$$
\begin{aligned}
\operatorname{RMS}\left(D_{N}\right) & =\frac{\sigma_{\eta}}{\sqrt{N}}, \\
& =\frac{\sigma_{\eta}}{\sqrt{T f_{s}}} .
\end{aligned}
$$

By integrating the above average value over $T$, the standard deviation of the integral of noise at time $T$, denoted as $S_{v}(T)$, can be obtained:

$$
\begin{aligned}
S_{v}(T) & =\int_{0}^{T} D_{N} d t \\
R M S\left(S_{v}(T)\right) & =\int_{0}^{T} R M S\left(D_{N}\right) d t \\
& =\sigma_{\eta} \frac{\sqrt{T}}{\sqrt{f_{s}}} .
\end{aligned}
$$

Thus, for the case in which a bound for $\eta^{<1>}$, cannot be given a priori but there is some knowledge of the bounds of noise we propose to use a time varying $\delta$ in algorithm A1 defined as follows

$$
\begin{aligned}
\eta^{<1>}(T) & =4 R M S\left(S_{v}(T)\right) \\
& =4 \sigma_{\eta} \frac{\sqrt{T}}{\sqrt{f_{s}}}
\end{aligned}
$$

The reason for the above criterion is that $P\left(\left|S_{v}(T)\right| \leq 4 R M S\left(S_{v}(T)\right)\right) \approx 0.99994$, giving a good reliability that the drift due to noise integration will be below such value. The time $T$ of integration is evaluated as

$$
T=t-t_{0}+f_{s}^{-1}
$$

the term $f_{s}^{-1}$ is introduced for practical reasons, in this way in $\delta$ is considered at least one sampling time of integration.

Remark 6: Instead of the standard deviation $\sigma_{\eta}$, which may be difficult to know, and in order to overestimate the threshold $\eta^{<1>}$, we propose to use an upper bound bound of the noise as $\sigma_{\eta}$. Thus, using $\sigma_{\eta}=\bar{\eta}^{<0>}$ and 49 ) in (48), the expression (18) can be obtained.

\section{References}

Bernardin, S. L. (2006, March). Wavelet processing for pitch period estimation. In Proceedings of the 38th southeastern symposium on system theory. Cookeville, TN, USA.

Dempster, A. P., Laird, N. M., \& Rubin, D. B. (1977). Maximum likelihood from incomplete data via the EM algorithm. Journal of the Royal Statistical Society, Series B, 39(1), 1-38.

Estrada, A., Efimov, D., \& Perruquetti, W. (2014, August). Position and velocity estimation through acceleration measurements. In 19th world congress of the international federation of automatic control (p. 6460-6465). Cape Town, South Africa.

Feder, M. (1993). Parameter estimation and extraction of helicopter signals oberved with a wide-band interference. IEEE Transactions on Signal Processing, $41(1), 232-244$.

Grewal, M. S., Weill, L. R., \& Andrew, A. (2007). Global positioning, inertial navigation and integration (2nd ed.). John Wiley \& Sons.

Guangyu, K., \& Shize, G. (2009). Improving AMDF for pitch period detection. In International conference on electronic measurement and instruments.

Karreman, G., \& Prood, C. (1995). Heart muscle contraction oscillation. International Journal of Bio-Medical Computing, 38, 49-53.

Liu, T., Inoue, Y., \& Shibata, K. (2009). Development of a wearable sensor system for quantitative gait analysis. Measurement, 42, 978-988. 
Min, Y., Yingchun, Y., \& Zhaohui, W. (2005). A pitched-based rapid speech segmentation for speaker indexing. In Seventh ieee international symposium on multimedia.

Peeters, G. (2006, May). Music pitch representation by periodicity measures based on combined temporal and spectral representations. In Icassp 2006 proceedings. ieee international conference on acoustics, speech and signal processing (Vol. 5, p. V).

Riviere, C. N., Ang, W. T., \& Khosla, P. K. (2003). Toward active tremor canceling in hanheld microsurgical instruments. IEEE Transactions on Robotics and Automation, 19, 793-800.

Rogers, R. M. (2003). Applied mathematics in integrated navigation systems (J. A. Schetz, Ed.). Reston : American Institute of Aeronautics and Astronautics, Inc.

Schepers, H. M., Asseldonk, E. H. van, Baten, C. T., \& Veltnik, P. H. (2010). Ambulatory estimation of foot placement during walking using inertial sensors. Journal of biomechanics, 43, 3138-3143.

Spence, G., \& Clarke, I. (2000). A time-domain analysis of multiple periodic signals. In Iee seminar on time-scale and time-frequency analysis and applications (p. 2/1-2/10). London.

Thong, Y., Woolfson, M., Crowe, J., Hayes-Gill, B., \& Challis, R. (2002). Dependence of inertial measurement of distance on accelerometer noise. Measurement Science and Technology, 13(8), 1163 1172.

Thong, Y., Woolfson, M., Crowe, J., Hayes-Gill, B., \& Jones, D. (2004). Numerical double integration of acceleration measurements in noise. Measurement, 36(1), 73 - 92.

Veluvolu, K. C., \& Ang, W. T. (2011). Estimation of physiological tremor from accelerometers for real-time applications. Sensors (11), 3020-3036. 